\title{
Similarity, Topology, and Physical Significance in Relativity Theory
}

\author{
Samuel C. Fletcher*
}

\begin{abstract}
Stephen Hawking, among others, has proposed that the topological stability of a property of spacetime is a necessary condition for it to be physically significant. What counts as stable, however, depends crucially on the choice of topology. Some physicists have thus suggested that one should find a canonical topology, a single 'right' topology for every inquiry. While certain such choices might be initially motivated, some littlediscussed examples of Robert Geroch and some propositions of my own show that the main candidates - and each possible choice, to some extent - faces the horns of a no-go result. I suggest that instead of trying to decide what the 'right' topology is for all problems, one should let the details of particular types of problems guide the choice of an appropriate topology.
\end{abstract}

\section{Introduction}

There are many reasons to consider notions of similarity in philosophy and, more specifically, philosophy of science. They underlie Lewis's famous system for counterfactual semantics, modal logic, laws of nature and causation ([1973]; [1986]). More recently, Halvorson ([2012]) has suggested that similarity is salient for characterizing scientific theories formally: one cannot recover a theory from its models unless one encodes the similarity amongst the models' truth-valuations topologically. And in philosophy of physics, Manchak ([unpublished]) has remarked that placing a topology on the models of general relativity can describe how different possible relativistic worlds (i.e., relativistic spacetimes) are 'nearby' one another.

This topological approach to similarity has been used by physicists working in mathematical relativity since the 1970s. In this context the notion of stability has been crucial. Roughly, a property of an object $O$ of a specified class (like mathematical models, solutions to a differential equation, etc.) is stable when all objects in that class sufficiently similar to $O$ also have that property - the name 'stability' comes from the intuitive picture that the

*Thanks to three referees from the British Journal for Philosophy of Science and to audiences at Irvine and Pittsburgh for helpful comments, especially Jeff Barrett, Ben Feintzeig, Dennis Lehmkuhl, David Malament (who inquired about corollary 2), John Norton, and Chris Wüthrich, and to Jim Weatherall for much guidance, including suggesting proposition 3 and sketching part of proposition 4. John Manchak spurred me to generalize a special case of proposition 5. Part of the research leading to this work was completed with the support of a National Science Foundation Graduate Research Fellowship. 
property is preserved under arbitrary (but sufficiently small) perturbations. In this paper I am interested in exploring how this literature connects similarity - in particular, stabilitywith judgments of physical significance. For example, Hawking has asserted that 'the only properties of space-time that are physically significant are those that are stable in some appropriate topology' ${ }^{1}$ and that '[f] or physical purposes it is sufficient to prove that a theorem holds generically' ([1971], p. 395).

One attraction of Hawking's proposal is that, given an 'appropriate topology', it would seem to reduce part of a philosophical question to a technical question: in certain cases, instead of puzzling over whether a property is 'physically significant', one instead may determine on which sets it is unstable; instead of assessing the import of apparent isolated counterexamples to a theorem, one simply proves that the theorem holds generically. Whether this reduction is successful, however, depends first on justifying why there should be a connection between physical significance and topological stability at all. Then, given a satisfactory justification, one must explicate what an 'appropriate topology' is supposed to be. After addressing the former query in $\S 2$, I focus on the latter in the remainder. Since a choice of topology on spacetimes encodes particular ways in which those spacetimes are similar, an appropriate topology is one that gets this notion of similarity right. While it would greatly simplify matters if there were a canonical such topology, in $\S 3-4$ I consider two classes of topologies often considered in the literature and find them flawed for this purpose. (The propositions I prove to argue this point may be of independent foundational interest.) Against suggestions from some physicists, in $\S 5$ I suggest instead that there cannot be a canonical topology, and that an 'appropriate' topology must covary with the context of inquiry. Without a canonical topology, however, stability itself does not directly settle any conceptual questions about what is physically significant. Rather, whether a property counts as physically significant in a model depends upon the prior choice of topology - how one considers models to be relevantly similar.

\section{Similarity, Topology, and Physical Significance}

What does it mean to say that a property is physically significant? There is not likely any straightforward univocal concept that wholly underlies the broad use of this phrase in the physics literature, but it will suffice here to draw out some of its connections with epistemic warrant and stability. ${ }^{2}$ Physicists use mathematical models to represent physical phenomena, including past observations and potential predictions. But they must be judicious in using models to make inferences about phenomena, as models are often idealized or only approximate. 'Physical significance' expresses an aspect of this partial connection between models and phenomena. One says that a property of a model of physical phenomena is physically significant to the degree to which one has warrant to infer that property about the physical phenomena. For instance, one would say that the past singularity of the standard cosmological model is physically significant to the extent that one can infer about the actual universe that there is a past singularity, the Big Bang.

\footnotetext{
${ }^{1}$ See also (Geroch [1971], p. 70) and (Hawking and Ellis [1973], p. 197).

${ }^{2}$ I take myself to be making some tentative suggestions rather than undertaking any robust project of conceptual analysis.
} 
The connection with stability becomes evident in considering that the observed data used to build such models, based on quantities like length, energy and position, are typically imprecise. Imprecise data usually yield imprecise models, or rather a range of models compatible with the data's imprecision. For convenience, however, scientists typically build a single model and represent the imprecision in other ways. But the inferences warranted through that model must cohere with the inferences one would have made through the range of models - that is, these inferences must be compatible not just with the observed data, but also with the whole set of data values falling within the measurements' range of imprecision. Thus any inferences that crucially depend on perfectly precise data will never be warranted. Requiring the stability of a property as a necessary condition for its physical significance enforces this compatibility between imprecise data and the inferences one draws: the only properties about phenomena one can infer from a model are those that all arbitrarily similar models share. ${ }^{3}$

Returning to the example above, the data cosmologists have of the universe is imprecise, with a range of compatible cosmological models, including the standard one and a neighborhood of models similar to it. The standard model has a past singularity, and so one may inquire whether one has warrant to infer about the actual universe that there was a Big Bang. The requirement of stability would demand for such an inference that some neighborhood of cosmological models similar to the standard one also have a past singularity. For, one might argue, the data available is compatible with at least some such neighborhood, and any inferences about the world one draws should not depend on which data-compatible model one chooses. Things seem to turn out fortunately for the conclusions of standard cosmology, as there is in fact a sense in which the past singularity of the standard cosmological model is stable. ${ }^{4}$

It is important to contrast the epistemological character of physical significance as such with the more metaphysical character of being 'physically (un)reasonable' (Smeenk and Wüthrich [2011]; Manchak [2011]; Earman [1995], ch. 3.4) or just plain '(un)physical' (Norton [2008], §3.2). Although physicists do not usually explicitly distinguish these, they tend to say that a model is physically unreasonable when they wish to exclude it as a genuine physical possibility countenanced by a theory. By contrast, physical significance tends to refer to the warrant to infer properties of physical phenomena from a model. There are interesting connections to explore between the physically significant and the physically reasonable, ${ }^{5}$ but the following discussion shall focus on the former.

In order to apply the stability criterion for physical significance precisely, one should formalize the notion of similarity it depends upon. Topology is a natural choice. ${ }^{6}$ A topology on a class of objects determines notions of convergence and continuity, respectively, for

\footnotetext{
${ }^{3}$ Stability plays an analogous role in securing inference under idealization. Scientists often use idealized models to make inference more tractable. In these cases, one would like to infer properties of phenomena the de-idealized model represents from those of the idealized model. Even if the idealization is not too severe, such an inference will not be warranted unless the property in question is stable.

${ }^{4}$ Specifically, the property of the standard cosmological model that each inextendible timelike geodesic is past incomplete is stable in the $C^{2}$ open topology (Lerner [1973], theorem 6.1) introduced in $\S 3$.

${ }^{5}$ See, for example, (Fletcher [2012], §3.1) for such connections in the case of excluding the indeterministic trajectories of Norton's dome ([2008]).

${ }^{6}$ Many other choices, like uniform spaces, are strictly stronger than a topology, so one expects at least to reckon with topological structure.
} 
sequences and parameterized families of such objects, using its system of open neighborhoods to encode a weak sense of similarity. A property $P$ of an object $O$ (or, more generally, of each of a set of objects $\left\{O_{\alpha}\right\}$ ) is then stable just in case there is an open set containing $O$ (resp. $\left\{O_{\alpha}\right\}$ ), all of whose members also have $P .{ }^{7}$ Further, property $P$ holds generically on a collection $S$ when it holds on an open subset of $S$ that is dense in $S$. Density ensures that elements of $S$ that do not have $P$ do so unstably, and openness ensures that elements that do have $P$ do so stably.

However, if the collection of objects is infinite, as is the case with relativistic spacetimes, then there will be infinitely many topologies one can place on the collection, topologies that can differ regarding whether a property is stable or generic on a subcollection. How can one decide which topology is appropriate? ${ }^{8}$ Perhaps there is in fact a canonical topology: a single choice of topology over the collection of relativistic spacetimes that should apply whenever such a topology is needed. Such a position had at one time been suggested by Geroch, who writes, 'It is important, I feel, that one settles on one (or possibly two) topologies in which to work rather than discovering a new topology for each new theorem' ([(1970], p. 269) ${ }^{9}$ and more strongly by Lerner ([(1972], [1973]) and Lerner and Porter, who advocate for a particular choice: "if one regards all Lorentz metrics on $M$ as being on an equal (mathematical $[s i c]$ footing, it appears that the only acceptable choice for a topology is the Whitney fine $C^{k}$ topology' ([1974], p. 1413). Those familiar with Lewis's ([1986]) influential (albeit controversial) analysis of comparative similarity for possible worlds based on laws of nature and particular matters of fact might also wonder if a similar general analysis might be given for relativistic spacetimes. In the remainder I investigate the viability of this canonicalism about topologies over spacetime, considering Lerner's choice in $\S 3$ and another initially plausible class of candidates in $\S 4$. In examining the problems each faces, the case against canonicalism will emerge.

\section{The Open Topologies}

Recall that a relativistic spacetime is an ordered pair $\left(M, g_{a b}\right)$, where $M$ is a four-dimensional smooth manifold ${ }^{10}$ and $g_{a b}$ is a smooth Lorentzian metric on $M$, whose indices are abstract. ${ }^{11}$ Then the collection of objects to topologize consists of the Lorentzian metrics on a fixed

\footnotetext{
${ }^{7}$ There is another related sense of stability associated with the initial value problem in general relativity: one says that the evolution of initial data on a Cauchy surface is stable when its map into the evolved relativistic spacetime is continuous (Hawking and Ellis [1973], p. 253). Because this requires considering only globally hyperbolic spacetimes, here I focus on the 'geometric' version of stability.

${ }^{8}$ While the same question arises for topologies on finite collections of objects, in that case one might hold out hope to be able to decide by direct comparison.

${ }^{9}$ I do not attribute to him outright advocacy, since a careful reading reveals an admixture of methodological pragmatism: 'I think it is important [...] to eventually settle on one or possibly two topologies with which to work. Hardly any economy of thought results if there are hundreds of topologies in use' ([1971], p. 73). Moreover, later writings indicate a preference for the methodologically contextualist approach I take in $\S 5$ : 'The topology one chooses in practice depends on what one wants the topology to do' ([1985], pp. 175-6).

${ }^{10}$ One also requires $M$ to be connected, paracompact, and Hausdorff.

${ }^{11}$ That is, the super- and subscripts of tensor fields like $g_{a b}$ label copies of vector spaces in which the fields reside. See, e.g., (Malament [2012], §1.4).
} 
manifold $M$, which I denote $L(M) .{ }^{12}$ The Whitney fine $C^{k}$ topology, also called the $C^{k}$ open topology, may then be defined as follows. First, let $h^{a b}$ be some smooth Riemannian (inverse) metric on $M$, and define the 'distance' function between the $k^{\text {th }}$ partial derivatives of two Lorentz metrics $g_{a b}$ and $g_{a b}^{\prime}$, relative to $h^{a b}$ and at each point of $M$, as the scalar field

$$
d\left(g, g^{\prime} ; h, k\right)= \begin{cases}{\left[h^{r u} h^{s v}\left(g_{r s}-g_{r s}^{\prime}\right)\left(g_{u v}-g_{u v}^{\prime}\right)\right]^{1 / 2},} & k=0, \\ {\left[h^{a_{1} b_{1}} \cdots h^{a_{k} b_{k}} h^{r u} h^{s v}\right.} & k>0, \\ \left.\otimes \nabla_{a_{1}} \cdots \nabla_{a_{k}}\left(g_{r s}-g_{r s}^{\prime}\right) \nabla_{b_{1}} \cdots \nabla_{b_{k}}\left(g_{u v}-g_{u v}^{\prime}\right)\right]^{1 / 2}, & \end{cases}
$$

where $\nabla$ is the Levi-Civita derivative operator compatible with $h_{a b}$. I have omitted the abstract indices in the arguments of $d$ since they needlessly clutter the notation, and I will hereafter continue to drop them when they will never be contracted.

The function $d\left(g, g^{\prime} ; h, k\right)$ compares $g$ and $g^{\prime}$ at each point of $M$ through the Euclidean distance between the components of their $k^{\text {th }}$ order partial derivatives as determined by $h$. Then the sets of the form

$$
B_{k}(g, \epsilon ; h)=\left\{g^{\prime}: \sup _{M} d\left(g, g^{\prime} ; h, 0\right)<\epsilon, \ldots, \sup _{M} d\left(g, g^{\prime} ; h, k\right)<\epsilon\right\}
$$

constitute a basis for the $C^{k}$ open topology, where $g$ ranges over all Lorentz metrics, $\epsilon$ ranges over all positive constants, ${ }^{13}$ and $h$ ranges over all Riemannian (inverse) metrics. One can view these basis elements as generalizations of the $\epsilon$-balls familiar to metric spaces.

But how does one justify the $C^{k}$ open topology as canonical? For instance, how should one choose the right value of $k$ ? One way is to investigate examples of stability about which one has a strong intuition, ruling out available topologies that do not meet them. For example, in discussing a theorem proving the stability of the strong energy condition ${ }^{14}$ in the $C^{2}$ open topology, Lerner writes,

It should be pointed out that [this theorem] is not true in any of the weaker topologies frequently used [...]. If we agree that any reasonable topology [...] should allow perturbations preserving the existence of non-zero rest mass, we may take this as further evidence in favor of the [open] topologies.

(Lerner [1973], p. 28)

Indeed, it seems that virtually all of the results regarding stability and genericity of global properties of spacetimes have used one of the open topologies. For example, the encyclopedic monograph Global Lorentzian Geometry, which has an entire chapter on 'stability of [geodesic] completeness and incompleteness', defines only the open topologies for these purposes (Beem et al. [1996], p. 63 \& ch. 7).

However widely accepted, the universal appropriateness of the open topologies has not gone unquestioned. Geroch ([(1970], [1971]) has provided a pair of examples that illustrate

\footnotetext{
${ }^{12}$ One might of course also wish to compare spacetimes whose underlying manifolds are not diffeomorphic. Although Hawking and Ellis ([1973], p. 198) state that this can be done, to my knowledge no one has done so nontrivially for all spacetimes.

${ }^{13}$ One can choose a different $\epsilon$ for each derivative order, but the resulting basis generates the same topology.

${ }^{14}$ This is the condition that for any timelike vector $\xi^{a}$ at any point of $M,\left(T_{a b}-\frac{1}{2} T g_{a b}\right) \xi^{a} \xi^{b} \geq 0$, where $T_{a b}$ is the stress-energy tensor and $T$ is its trace. See (Hawking and Ellis [1973], p. 95) or (Malament [2012], p. 166).
} 
some surprising features of the $C^{0}$ open topology in particular. ${ }^{15}$ His first example is a sequence that seems like it should converge to Minkowski spacetime but in fact does not. Explicitly, the sequence of metrics

$$
\stackrel{m}{g}_{a b}=\left(1+\frac{1}{m^{2}+x^{2}+y^{2}+z^{2}}\right)\left(d_{a} t\right)\left(d_{b} t\right)-\left(d_{a} x\right)\left(d_{b} x\right)-\left(d_{a} y\right)\left(d_{b} y\right)-\left(d_{a} z\right)\left(d_{b} z\right)
$$

on $\mathbb{R}^{4}$, where $t, x, y, z$ are scalar coordinate fields, does not converge as $m \rightarrow \infty$ to the Minkowski metric

$$
\eta_{a b}=\left(d_{a} t\right)\left(d_{b} t\right)-\left(d_{a} x\right)\left(d_{b} x\right)-\left(d_{a} y\right)\left(d_{b} y\right)-\left(d_{a} z\right)\left(d_{b} z\right)
$$

even though the 'bump,' remaining centered at the coordinate origin, decreases in amplitude to zero. This is because $\stackrel{m}{g} \rightarrow \eta$ in the $C^{0}$ open topology if and only if for every neighborhood of the form $B_{0}(\eta, \epsilon ; h)$, we have $\stackrel{m}{g} \in B_{0}(\eta, \epsilon ; h)$ for $m$ sufficiently large. But one can always pick an $h$ growing sufficiently rapidly towards infinity that, for all $m, \sup _{M} d(\eta, \stackrel{m}{g} ; h, 0)=\infty$.

Geroch's second example is the one-parameter family

$$
\Lambda=\left\{\lambda g_{a b}: \lambda>0\right\}
$$

with a fixed $g_{a b}$ on a non-compact $M$, which strikingly does not trace out a continuous curve in the $C^{0}$ open topology-indeed, it is everywhere discontinuous. To see this, note that the family is continuous in the $C^{0}$ open topology if and only if for every $\lambda_{0}>0$ and every neighborhood of the form $B_{0}\left(\lambda_{0} g, \epsilon ; h\right)$, there is a positive open interval $I \ni \lambda_{0}$ such that $\left\{\lambda g_{a b}: \lambda \in I\right\} \subseteq B_{0}\left(\lambda_{0} g, \epsilon ; h\right)$. But, as with the first example, one can pick an $h$ growing sufficiently rapidly and without bound-recall $M$ is non-compact-so that for any $\delta \neq 0$, $\sup _{M} d\left(\lambda_{0} g,\left(\lambda_{0}+\delta\right) g ; h, 0\right)=\infty$.

This example is particularly surprising because the elements of $\Lambda$ have the same representational capacities - each can represent precisely the same class of spacetimes, and one can interpret the parameter $\lambda$ as a mere change of units. ${ }^{16}$ In fact, one can prove quite general results regarding the conditions under which a sequence converges or a family is continuous in the open topologies. Specifically, the following is sketched by Golubitsky and Guillemin ([1973], pp. 43-4):

Proposition 1. Let $g,\{\stackrel{n}{g}\}_{n \in \mathbb{N}}$ be Lorentz metrics on a non-compact manifold $M$. Then $\stackrel{n}{g} \rightarrow g$ in the open $C^{k}$ topology on $L(M)$ iff there is a compact $C \subset M$ such that:

1. for sufficiently large $n, \stackrel{n}{g}_{\mid M-C}=g_{\mid M-C}$; and

2. $\stackrel{n}{g}_{\mid \operatorname{int}(C)} \rightarrow g_{\mid \operatorname{int}(C)}$ in the open $C^{k}$ topology on $L(\operatorname{int}(C))$.

\footnotetext{
${ }^{15}$ In fact, they work just as well for any of the $C^{k}$ open topologies but I follow Geroch in presenting them in the $C^{0}$ context where the calculations are simplest.

${ }^{16}$ It also demonstrates that the open topologies, like the other topologies that I will consider, 'overrepresent' the Lorentz metrics on $M$ since in general they represent isometric spacetimes through distinct points. One can compensate for this defect somewhat by ensuring one constructs only invariant topologies (Geroch [(1970], pp. 281-2), ones for which the pushforward map induced by any element of the diffeomorphism group of $M$ acts on $L(M)$ as a homeomorphism. Indeed, all of the topologies considered in this paper are invariant in this way.
} 
In other words, a sequence converges in the $C^{k}$ open topology just in case its elements eventually equal the limit point everywhere except at most on (the interior of) a compact set, a criterion of convergence even stronger than uniform! One can then use this proposition to prove (see $\S$ A.1) a necessary condition for a family of Lorentz metrics to be continuous.

Proposition 2. Suppose that $L(M)$ is given the $C^{k}$ open topology, with $M$ non-compact. If $f: \mathbb{R} \rightarrow L(M)$ is continuous, then for every $x_{0}, x_{1} \in \mathbb{R}$, there is some compact $C \subset M$ such that $f\left(x_{0}\right)_{\mid M-C}=f\left(x_{1}\right)_{\mid M-C}$.

Thus any pair from a continuous one-parameter family of Lorentz metrics must always be equal everywhere except at most on a compact set. Intuitively, one might picture the difference between the members of such a pair as a 'bump in a rug' that the function $f$ pushes around. Although the bump may be bigger or smaller, wider or narrower, it always has compact support. This is clearly a quite restricted class of continuous families. For example, because any two distinct members from the scale family of metrics given by eq. 5 are equal nowhere, proposition 2 ensures that this family is everywhere discontinuous.

One might object that the discussion of canonicalism was motivated by considering the connection of stability with physical significance, but Geroch's examples and propositions 1 and 2 bear on convergence and continuity. Why should problems with the latter bear on the use of topology for the former? Two responses are on offer. First, if a canonicalist would maintain this objection while affirming that continuity and convergence are worthy of investigation, ${ }^{17}$ she would already concede her position in suggesting that different purposes - stability, as opposed to convergence and continuity - may require different topologies. Second, convergence, continuity and stability are all interdependent, each determined by a topology's lattice of open sets. Recall, for instance, that the stability of a property depends on the existence of a certain open set. Thus it is in a sense easier for a property to be stable in a finer topology, since there are more open sets available that could be witnesses to stability. In particular, if a property is stable on a certain set in a given topology $\mathcal{T}$, it is stable in every topology finer than $\mathcal{T}$. Recall as well that the convergence of a sequence depends on certain aspects of every open neighborhood of its purported limit point. Thus it is in a sense easier for a sequence to converge in a coarser topology, since there are fewer open sets that must fulfill the proper role. In particular, if a sequence converges in a given topology $\mathcal{T}$, it converges in every topology coarser than $\mathcal{T}$.

Geroch's examples and propositions 1 and 2 therefore suggest that there are more open sets in the open topologies than one might have initially thought, which would perhaps make stability too easily achieved. For example, if a property is stable for $g$ in the $C^{0}$ open topology on $L(M)$ for non-compact $M$, then it obtains on some basic neighborhood $B_{0}(g, \epsilon ; h)$. However, for any $g^{\prime} \in B_{0}(g, \epsilon ; h)$ distinct from $g$ on a non-compact set, by choosing $h^{\prime}=\Omega h / d\left(g, g^{\prime} ; h, 0\right)$ for some unbounded $\Omega$, there is no $\epsilon^{\prime}$ for which $g^{\prime} \in B_{0}\left(g, \epsilon^{\prime} ; h^{\prime}\right)$. But one might think that whether $g^{\prime}$ can be sufficiently close to $g$, for some standard of sufficiency, should not depend on the choice of $h$, which is simply a device for comparing $g$

\footnotetext{
${ }^{17}$ Lerner ([(1972], [1973]) is aware of Geroch's examples and propositions 1 and 2, but concludes from them that one must give up talking about continuity and convergence. Considering that topology is typically introduced (in part) in the first place to treat continuity and convergence, a more measured response would be to reject the open topologies as canonical.
} 
and $g^{\prime}$. That the open topologies do have this dependence is ultimately what is responsible for propositions 1 and $2 .^{18}$

\section{Continuity in the Geometric Sense and the Compact- Open Topologies}

Given the problems that the open topologies face, one might very well abandon Lerner's suggestion and investigate other possible choices for a canonical topology. One idea comes from Geroch ([1969]), who has proposed a way of interpreting certain limiting relations entirely geometrically through the continuity (smoothness, etc.) of certain fields. Roughly, in the simplest case of a one-parameter family, one constructs a 5-dimensional manifold from the 4-dimensional manifolds of the family 'stacked' by their identifying parameter.

More precisely, suppose that one is given a family of metrics $\left\{\stackrel{t}{g}_{a b}\right\}_{t \in \mathbb{R}}$ on a fixed manifold $M .^{19}$ Let $\mathcal{M}$ be a manifold diffeomorphic to $M \times \mathbb{R}$ and let $\psi^{(t)}: M \rightarrow \mathcal{M}$ be a family of embeddings defined, using this diffeomorphism, by $p \mapsto(p, t)$. Thus the field $\tilde{t}: \mathcal{M} \rightarrow \mathbb{R}$ defined by $(p, t) \mapsto t$ is smooth and labels the 4-dimensional hypersurfaces foliating $\mathcal{M}$ by their parameter value $t$. Then one can define a symmetric field $\Gamma^{a b}$ on $\mathcal{M}$ with signature $(+,-,-,-, 0)$ by stipulating that $\left(\Gamma^{a b}\right)_{\mid(p, t)}=\left(\psi_{p}^{(t)}\right)_{*}\left({ }^{t} g^{a b}\right)$. In other words, $\Gamma^{a b}$ is the field that on each $\tilde{t}$-constant hypersurface is just the pushforward of the inverse Lorentz metric $\stackrel{t}{g}^{a b}$. One can find a fixed derivative operator $\nabla$ that is compatible with $\Gamma^{a b}$ and $\nabla_{a} \tilde{t}$, i.e., $\nabla_{a} \Gamma^{b c}=\mathbf{0}$ and $\nabla_{b} \nabla_{a} \tilde{t}=\mathbf{0}$, and that makes these two fields orthogonal, i.e., $\Gamma^{a b} \nabla_{a} \tilde{t}=\mathbf{0} .^{20}$

Now, for each $p \in M$ the points $\psi^{(t)}(p)$ for all $t$ form a smooth curve and the collection of all such curves for all $p$ form a congruence on $\mathcal{M}$ that indicates which points on different $\tilde{t}$-constant hypersurfaces are counterparts. Thus there is a vector field $\tau^{a}$ on $\mathcal{M}$ tangent to the curves of this congruence satisfying $\tau^{a} \nabla_{a} \tilde{t}=1$. This allows one at last to define a unique symmetric field $\Gamma_{a b}$ such that $\Gamma_{a b} \tau^{a}=\mathbf{0}$ and $\Gamma_{a b} \Gamma^{b c}=\delta_{a}^{c}-\tau^{c} \nabla_{a} \tilde{t}^{21}$ With this construction in place, we can say that the family $\stackrel{t}{g}_{a b}$ on $M$ is continuous in the geometric sense when the corresponding field $\Gamma_{a b}$ is continuous everywhere on $\mathcal{M}$. (Analogous definitions would apply to smoothness, etc.) One can similarly define the limit of a sequence of metrics by embedding the sequence in a one-parameter family.

A great appeal of this proposal is that it uses the natural, widely accepted geometrical formulation of a relativistic spacetime to do the work of choosing the canonical topology. On its face it does not seem to involve the kind of arbitrary decisions used in selecting an

\footnotetext{
${ }^{18}$ Like with Geroch's examples, eqs. 3 and 5 , this dependence applies for all the open topologies. I've only presented the $C^{0}$ case to illustrate it economically.

${ }^{19}$ Geroch does not require that the metrics be defined on diffeomorphic manifolds, but I can confine attention to that case here.

${ }^{20}$ For those familiar with the geometrized formulation of Newtonian gravitation, $\nabla_{a} \tilde{t}$ functions much like the temporal metric and $\Gamma^{a b}$ like the spatial metric, except that the latter is Lorentzian instead of Riemannian and only assumed to be smooth on each $\tilde{t}$-constant hypersurface. I would like to thank Jim Weatherall for emphasizing this point to me.

${ }^{21}$ This also parallels the construction of the covariant spatial metric in geometrized Newtonian gravitation. Cf. fn. 20 and (Malament [2012], p. 254, proposition 4.1.12).
} 
open topology. It turns out that the topology determined by all the families continuous in the geometric sense is well-known:

Proposition 3. A family of Lorentz metrics $\{\stackrel{t}{g}\}_{t \in \mathbb{R}}$ is continuous in the geometric sense iff it is continuous in the $C^{0}$ compact-open topology. ${ }^{22}$

(For a proof, see $\S$ A.2.) A basis for the $C^{k}$ compact-open topologies, for any non-negative integer $k$, may be written as sets of the form

$$
B_{k}(g, \epsilon ; h, C)=\left\{g^{\prime}: \sup _{C} d\left(g, g^{\prime} ; h, 0\right)<\epsilon, \ldots, \sup _{C} d\left(g, g^{\prime} ; h, k\right)<\epsilon\right\},
$$

where $g$ ranges over all Lorentz metrics, $\epsilon$ ranges over all positive constants, $h$ ranges over all Riemannian (inverse) metrics, ${ }^{23}$ and $C$ ranges over all compact subsets of $M$. The essential difference between the open and the compact-open topologies is that the former 'control' behavior everywhere on the manifold whereas the latter do so only on compact subsets.

Notably, one can show that, unlike with the open topologies, the sequence defined by eq. 3 converges to the Minkowski metric and the family defined by eq. 5 is continuous relative to the $C^{k}$ compact-open topologies. These topologies are also attractive for having a number of other interesting features. First, they coincide with the topology of $C^{k}$ compact convergence - that is, a sequence of metrics $\stackrel{n}{g} \rightarrow g$ on $M$ just when it and its partial derivatives to order $k$ (with respect to the Levi-Civita derivative operator compatible with an arbitrary Riemannian metric on $M$ ) do so uniformly on each compact $C \subseteq M$ (Munkres [2000], p. 283, theorem 46.2). ${ }^{24}$ Second, if a sequence of $C^{k}$ metrics $\stackrel{n}{g}$ converges to $g$, then $g$ is guaranteed to be at least $C^{k}$ as well (Munkres [2000], p. 284, corollary 46.6). Third, there is a close connection with homotopy. One can show that a family of Lorentz metrics is continuous in the $C^{k}$ compact-open topology if and only if it traces out a $C^{k}$ path in $L(M)$. So, in a way, the $C^{k}$ compact-open topology encodes which Lorentz metrics can be continuously (to order $k$ ) deformed into one another. ${ }^{25}$

Like with the open topologies, however, Geroch has criticized the general appropriateness of the compact-open topologies, contending that they rule counterintuitively on the sequence of metrics

$$
\stackrel{m}{g}_{a b}^{\prime}=\left(1+\frac{m}{1+(x-m)^{2}}\right)\left(d_{a} t\right)\left(d_{b} t\right)-\left(d_{a} x\right)\left(d_{b} x\right)-\left(d_{a} y\right)\left(d_{b} y\right)-\left(d_{a} z\right)\left(d_{b} z\right)
$$

on $\mathbb{R}^{4}$, where $t, x, y, z$ are natural scalar coordinate fields. ${ }^{26}$ "The "bump" in the metrics becomes larger as it recedes to infinity', he writes, but the 'sequence does approach Minkowski

\footnotetext{
${ }^{22}$ In particular, the $C^{0}$ compact-open topologies are the final topologies respectively associated with the families continuous in the geometric sense, the finest topologies on $L(M)$ that make those families continuous.

${ }^{23}$ Strictly speaking, letting $h$ range is superfluous, as the same topology is generated through a single choice (Geroch [(1970], p. 280 fn.).

${ }^{24}$ The compact-open topology coincides with the topology of compact convergence on a function space when the range of the functions is a metrizable space (Munkres [2000], pp. 285-6), and the bundle of Lorentz tensors over $M$, being a finite-dimensional manifold, is metrizable.

${ }^{25}$ Equivalently, the family is continuous in the $C^{k}$ compact-open topology just when the $k$-jets of the family belong to the same path component.

${ }^{26}$ The formula for the first term is garbled in (Geroch [1971], p. 71), but appears without error in (Geroch [(1970], p. 280).
} 
space in the $\left[C^{0}\right.$ compact-open $]$ topology (because the metrics become Minkowskian in every compact set)' ([1971], p. 71). In other words, since $d(\eta, \stackrel{m}{g} ; h, 0)$ is continuous for any choice of (smooth) Riemannian $h$, its supremum is bounded on any compact set and will become as small as one likes for sufficiently large $m$. However, '[i]ntuitively, we would not think of this sequence as approaching Minkowski space' ([1971], p. 71) (or presumably any spacetime at all). Thus he takes the $C^{0}$ compact-open topology to be too coarse.

This example is less convincing than his examples for the open topology. It is instructive to compare eq. 7 with the sequence of Taylor expansions of a real function like $\sin (x)$. For any particular finite-order expansion, one can find a sufficiently large $x$ such that the expansion, evaluated at this $x$, differs from $\sin (x)$ by as much as one wishes. But if one fixes some compact region of $\mathbb{R}$, then the Taylor series converges uniformly on that region. Similarly, the sequence given by eq. 7 converges to Minkowski spacetime because the $C^{0}$ compactopen topology corresponds with the topology of compact convergence. Just as the compact convergence of Taylor expansions seems perfectly reasonable, it is not clear why the same cannot be said in the case of sequences of Lorentz metrics.

However, there are other counterintuitive features of the compact-open topologies that bear even more directly on stability. For example, consider Hawking's theorem (Hawking and Ellis [1973], p. 198, proposition 6.4.9):

Theorem 1 (Hawking). The existence of a global time function on a relativistic spacetime is equivalent to stable causality, an absence of closed causal curves that is stable in the open $C^{0}$ topology.

If one of the compact-open topologies were to be canonical, one would want to know whether Hawking's theorem holds with respect to it as well. It turns out that it does not, and spectacularly so. In fact, according to the compact-open topologies, spacetimes generically contain closed timelike curves.

Proposition 4. Chronology violating spacetimes are generic in $L(M)$ in any of the $C^{k}$ compact-open topologies. ${ }^{27}$

Corollary 1. No Lorentz metric is stably causal in any of the $C^{k}$ compact-open topologies on $L(M) .^{28}$

In particular, according to the compact-open topologies, not only does Minkowski spacetime fail to be stably causal despite its global time function, it turns out that not having closed timelike curves is not physically significant! In other words, one would never have warrant to infer from a model of relativistic spacetime that the physical situation it repre-

\footnotetext{
${ }^{27}$ This slightly improves statements by Hawking ([1971], pp. 396-7) and Hawking and Ellis ([1973], p. 198), who advert without proof to the density of chronology violating spacetimes in $L(M)$ in any of the $C^{k}$ compact-open topologies.

${ }^{28}$ Cf. proposition 5.1 of (Manchak [unpublished]), which shows that each Lorentz metric is homotopic to one that violates chronology. As alluded to above, there is a close connection between homotopy and the compact-open topologies: the $C^{k}$ homotopy classes correspond with the path components of the $C^{k}$ compact-open topologies.
} 
sents does not permit a form of time travel. ${ }^{29}$ An alternative but equivalent definition of stable causality brings out why: a spacetime $(M, g)$ is stably causal with respect to the $C^{0}$ open topology just when there is a metric $g^{\prime}$ for which there are no closed causal curves and whose light cones everywhere lie outside those of $g$. By contrast, when stable causality is defined with respect to the $C^{0}$ compact-open topology, the light cones of $g^{\prime}$ need only lie outside of those of $g$ on a compact subset of $M$, leaving the rest unconstrained and ripe for the sprouting of closed causal curves.

By contrast, if $(M, g)$ already contains a closed timelike curve $\gamma: I \rightarrow M$, then one can pick a local basis element $B_{k}(g, \epsilon ; h, C)$ from any compact-open topology so that $\gamma[I] \subseteq C$ and $\epsilon$ is small enough so that, for any $g^{\prime} \in B_{k}(g, \epsilon ; h, C), \gamma$ is still $g^{\prime}$-timelike.

Proposition 5. Every spacetime containing a closed timelike curve does so stably in any of the $C^{k}$ compact-open topologies.

Thus one always has warrant to infer from spacetimes with closed timelike curves that they represent the possibility for a type of time travel. One ought also, for some relativistic spacetime models without closed timelike curves, have warrant to infer that they do not represent this possibility. That this never occurs under the compact-open topologies militates against taking any of them as canonical.

\section{Methodological Contextualism}

Any canonical topology on $L(M)$ should have the ability to properly distinguish which sequences converge, which families are continuous, and which properties are stable or generic. But as the previous two sections laid out, the two main classes of topologies in the literature fall short of these goals. The open topologies, advocated by Lerner, seem too fine to treat convergence and continuity. The compact-open topologies, naturally suggested through continuity in the geometric sense, seem too coarse for stable causality because their neighborhoods control behavior only on compact sets. Of course, that Geroch's examples do evince genuine problems for the former can well be challenged, and one may decide, according to one's inclinations, to bite the bullets of propositions 1 and 2, or 4 , but this does not completely resolve the issue of how to choose the canonical topology. Proponents of a canonical topology must decide without being ad hoc on which counterintuitive results to accept and are obliged to provide an explanation as to why the intuitive features thereby denied do not have the significance they seemed to.

The considerations already raised for the canonicalist can be cast in terms of a no-go result. Given some manifold $M$, proposition 3 entails that the $C^{0}$ compact-open topology on $L(M)$ is the finest topology in which all the one-parameter families continuous in the geometric sense are continuous. (See fn. 22.) But it follows from corollary 1 that no Lorentz metric is stably causal in the $C^{0}$ compact-open topology or any topology coarser than it. So if there is some Lorentz metric on $M$ that admits of a global time function, Hawking's

\footnotetext{
${ }^{29}$ This result does not state that one can have no inductive evidence for certain global properties, as one might interpret proposition 2 of (Manchak [2011], p. 414). Rather, it states that even if one had data for the whole universe, however imprecise, and fit that data to a relativistic spacetime, one could never conclude that there were no closed timelike curves in the universe if one takes any compact-open topology as canonical.
} 
theorem fails for it in such a topology. (Without the existence of such a metric, Hawking's theorem is vacuous.) This yields the following.

Proposition 6. If there is some Lorentz metric on $M$ that admits of a global time function, then there is no topology on $L(M)$ relative to which both

1. all one-parameter families continuous in the geometric sense are continuous, and

2. Hawking's theorem holds.

One might object that asking for such compatibility is too much; perhaps there is hope for compatibility with a weakened version of Hawking's theorem, in which one demands the existence of a global time function just in case the spacetime is stably causal when restricted to compact sets (or rather their interiors). But this fails, too.

Corollary 2. Suppose $\mathcal{T}_{M}$ is a topology on $L(M)$, for any $M$, that makes continuous all the one-parameter families continuous in the geometric sense. Then, for any $g \in L(M)$ there is no compact $C \subseteq M$ such that $g_{\mid \operatorname{int}(C)}$ is stably causal in the topology $\mathcal{T}_{\operatorname{int}(C)}$ on $L(\operatorname{int}(C))$.

Because there are no subsets of $M$ with the desired property, the analog of Hawking's theorem fails (as there remain many spacetimes with global time functions). ${ }^{30}$ This is just one of a possibly large family of 'no-go' results that the canonicalist must face.

But reminding oneself of the way these topologies are used suggests that one need not pick any canonical topology at all. Examining the consequences of adopting one topology over another is a part of the process of deciding which topology will be relevant for a given type of problem. Hawking has emphasized as much: 'A given property may be stable or generic in some topologies and not in others. Which of these topologies is of physical interest will depend on the nature of the property under consideration' ([1971], p. 396). Indeed, Geroch's later writings (see fn. 9) have indicated the same. If different topologies correspond to different ways one can specify how spacetimes are similar, it is not surprising that different topologies would be natural choices for different kinds of questions if those questions bear upon different kinds of properties. It thus seems best to accept a kind of methodological contextualism, where the best choice of topology is the one that captures, as best as one can manage, at least the properties relevant to the type of question at hand, ones that relevantly similar spacetimes should share. Thus, in contrast to the canonicalist, I would demand that particular choices of topology must be justified relative to a context as much as one feasibly can.

Methodological contextualism about topologies - at least in the sense of allowing oneself to pick the most appropriate topology for a given application instead of deciding on one in advance - would make all the above worries associated with picking a canonical topology moot. But the contextualist fortunately still has the resources to choose reasonable topologies - resources not so different from the canonicalists, but without the demand to

\footnotetext{
${ }^{30} \mathrm{In}$ fact, the stated weakening of stable causality with respect to the $C^{0}$ open topology is known to be equivalent to non-total imprisonment, the condition that no future-inextendible causal curve eventually enters but does not leave some compact subset of spacetime (Minguzzi [2009], theorem 1). That non-total imprisonment is much weaker than stable causality reveals that finding a weakening of stable causality that preserves Hawking's theorem is a subtle matter.
} 
select a single topology for all purposes. One will expect that similar questions will tend to demand similar topologies, so the process of justification need not be started afresh each time. In particular, one should arrive at a particular choice of topology through reflective equilibrium, balancing the demands of the current understanding of what different topologies capture physically and what notions of similarity one is trying to capture with the implications of new mathematical results, as the many examples and propositions of $\S 3-4$ did for the open and compact-open topologies. These new results may then change one's intuitions, which in turn may suggest further results to investigate. The more one can accumulate these kinds of facts, the more there will be relevant data at hand for a particular type of inquiry so that one can make a sharper, better justified conceptual decision regarding which topology to use. Sometimes this will lead one to reject initially promising and intuitive choices, and sometimes it will reinforce them. One need not postulate that this reflective equilibrium lead to a stable limit; even if one has accumulated many results in favor of using a particular topology for some narrow type of inquiry, one should still be open to new facts and connections that will disturb one's equilibrium. ${ }^{31}$

Nevertheless, more work needs to be done characterizing how particular choices of topology may be appropriate for a given kind of question. Consider again, for example, Hawking's stability criterion for physical significance. Per the discussion at the end of $\S 3$, one might expect that some topologies fine enough for the stability of some properties - like the open topologies - are too fine for certain sequences to converge, or vice versa - like the compactopen topologies. So the topologies that might be natural candidates for inquiries about physical significance through stability may be different from those for inquiries about continuity or convergence. ${ }^{32}$ At least in the case of stability, one may be able to characterize classes of properties to which particular topologies are (in)sensitive, or the range of topologies in which interesting properties, like stable causality, behave as one might expect. The usual classification of spacetime properties into local and global (Manchak [2011], p. 413) is too coarse for these purposes. ${ }^{33}$ Part of the difficulty in answering this question for stability - indeed, for any inquiry - stems from the small variety of topologies used in the literature. ${ }^{34}$ Theorems about the stability and genericity of global properties generally use the open topologies (e.g., see Hawking and Ellis ([1973], p. 198), Lerner ([1973]), and Beem et al. ([1996], ch. 7)). Theorems about the stability of Cauchy developments use variants on the coarser compact-open topologies (see Hawking ([1971], p. 398-9) and Hawking and Ellis ([1973], p. 252-254)). The-

\footnotetext{
${ }^{31}$ One can find this dynamic and non-teleological conception of reflective equilibrium in the literature on moral theorizing as well (Schroeter [2004]).

${ }^{32}$ I do not preclude there being different contexts in which the same kind of question ultimately demands a different topology. There may very well be different inquiries into physical significance, for example, that are best served by different choices.

${ }^{33}$ This classification takes a property $P$ of a spacetime $(M, g)$ to be local if and only if all spacetimes locally isometric to $(M, g)$ also have $P$, and global otherwise. Thus both the topology of $M$ and the existence of a closed timelike curve are global properties, whereas only the latter has any hope of having an analog to proposition 4.

${ }^{34}$ One pocket of innovation in this regard has been the community working on causal set theory, one proposal for a theory of quantum gravity. (For an introduction, see Henson ([2009], [2012]).) They have proposed topologies on $L(M)$ that treat the conformal and causal structure of spacetime separately, although some (Bombelli and Meyer [1989]) suffer problems similar to those of the open topologies, and others (Noldus [2002]) are restricted to globally hyperbolic spacetimes, hence are inappropriate for studying, e.g., the stability of causality conditions. A fuller discussion of these, however, lies outside the scope of this paper.
} 
orems concerning the convergence of relativistic spacetimes to Newtonian spacetimes (e.g., Malament ([1986])) have (implicitly) used a point-open topology, which is even coarser. ${ }^{35}$

It turns out that there is a simple modification to the $C^{0}$ open topology that makes the one-parameter families defined by eq. 5 everywhere continuous while, unlike the compactopen topology, still preventing the sequence defined by eq. 7 from converging. Take the basis elements of the $C^{0}$ open topology (eq. 2), but restricted only to bounded pairs $(g, h)$, ones for which $\sup _{M} d(g, \lambda g ; h, 0)<\infty$ for any positive $\lambda$. This prohibits choosing any $h$ that grows too rapidly, eliminating the open neighborhoods of each Lorentzian $g$ that forced eq. 5 to be everywhere discontinuous. One can show, moreover, that this topology lies between the open and compact-open topologies in coarseness. However the sequence defined by eq. 3 still does not converge to Minkowski spacetime according to this topology, so it still would not rule in the intuitively 'right' way according to Geroch.

But if further refinements are found that produce a topology satisfying Geroch's desiderata, might that topology end up being satisfactory for all demands? If I allow for the possibility that the methods available for picking an appropriate topology may eventually single out a unique choice, or perhaps very few, to what extent is methodological contextualism really distinguished from a slightly liberalized canonicalism? The answer is methodological. The two positions are not distinct because of differing ends - whether to use one topology or many-but because of their differing means: what grounds we might have to prefer one topology over another, and how those grounds need to be articulated. A canonicalist holds that because there are definitive reasons always to choose a single topology (or perhaps very few), there is no further reason to say why that choice is appropriate for a given type of inquiry. By contrast, the contextualist takes the relevant reasons to be provided by the type of problem at hand, not in advance, and that they should therefore be articulated and reasonably defended. It bears emphasizing that the latter does not deny that there can be principled reasons to pick out a certain topology, only that those reasons can ever be given in enough generality to preclude attention to the details of the type of situation at hand. We indeed be may be lucky for the sake of our economy of thought if a few topologies are always appropriate, but we should not obstruct the development of new ones if they fit particular purposes better.

\section{A Proofs of Propositions}

\section{A.1 Continuity and the open topologies}

Proposition 2. Suppose that $L(M)$ is given the $C^{k}$ open topology, with $M$ non-compact. If $f: \mathbb{R} \rightarrow L(M)$ is continuous, then for every $x_{0}, x_{1} \in \mathbb{R}$, there is some compact $C \subset M$ such that $f\left(x_{0}\right)_{\mid M-C}=f\left(x_{1}\right)_{\mid M-C} \cdot{ }^{36}$

Proof. The case where $f$ is a constant function is immediate, so suppose otherwise and pick arbitrary distinct $x_{0}, x_{1} \in \mathbb{R}$, assuming without loss of generality that $x_{0}<x_{1}$. I claim that,

\footnotetext{
${ }^{35}$ The point-open topologies are defined similarly to the compact-open topologies (eq. 6), but require that the suprema be taken over only finitely many points in each basis element instead of over compact sets.

${ }^{36}$ This proposition may be generalized to families of Lorentz metrics parameterized by any path-connected space.
} 
given any $r \in\left[x_{0}, x_{1}\right]$, there is an open (relative to $\left[x_{0}, x_{1}\right]$ ) interval $I_{r} \subseteq\left[x_{0}, x_{1}\right]$, containing $r$, such that for any $q \in I_{r}$, there is some compact $C(r, q) \subset M$ for which $f(r)_{\mid M-C(r, q)}=$ $f(q)_{\mid M-C(r, q)}$. For suppose otherwise, and consider any sequence of intervals $I_{r}^{1} \supset I_{r}^{2} \supset \ldots$ such that $I_{r}^{n} \subseteq\left[x_{0}, x_{1}\right]$ for each $n$ and $\bigcap_{n=1}^{\infty} I_{r}^{n}=\{r\}$. One can then construct by induction a sequence of metrics that converges to $f(r)$. For the base step, let $I_{r}^{s_{1}}=I_{r}^{1}$ and note that there is some $q_{1} \in I_{r}^{s_{1}}$ distinct from $r$ such that $f(r)_{\mid M-C} \neq f\left(q_{1}\right)_{\mid M-C}$ for any compact $C \subset M$. (Such a $q_{1} \neq r$ exists because $f$ is continuous.) For the inductive step, suppose $I_{r}^{s_{n}}$ is given so that there is some $q_{n} \in I_{r}^{s_{n}}$ distinct from $r$ such that $f(r)_{\mid M-C} \neq f\left(q_{n}\right)_{\mid M-C}$ for any compact $C \subset M$. Then pick some $I_{r}^{s_{n+1}}$ such that $s_{n+1}>s_{n}$ and $q_{n} \notin I_{r}^{s_{n+1}}$, noting that there is some $q_{n+1} \in I_{r}^{s_{n+1}}$ distinct from $r$ such that $f(r)_{\mid M-C} \neq f\left(q_{n+1}\right)_{\mid M-C}$ for any compact $C \subset M$. The induction is complete, so by construction the sequence $q_{n} \rightarrow r$ as $n \rightarrow \infty$, and for each $n, f(r)_{\mid M-C} \neq f\left(q_{n}\right)_{\mid M-C}$ for any compact $C \subset M$. But because $f$ is continuous, it follows that $f\left(q_{n}\right) \rightarrow f(r)$ as $n \rightarrow \infty$ (Munkres [2000], p. 130, theorem 21.3), and by proposition 1, this implies in turn that there is a compact $C \subset M$ for which $f(r)_{\mid M-C}=f\left(q_{n}\right)_{\mid M-C}$ for sufficiently large $n$, which is a contradiction.

Next, note that the $\left\{I_{r}: r \in\left[x_{0}, x_{1}\right]\right\}$ form an open cover of $\left[x_{0}, x_{1}\right]$ (relative to $\left[x_{0}, x_{1}\right]$ ). The interval is compact, so by definition there is some finite subcover $\left\{I_{r_{i}}: i=1, \ldots, m\right\}$, each of whose elements has, for all $q \in I_{r_{i}}$, an associated compact $C\left(r_{i}, q\right) \subset M$ for which $f\left(r_{i}\right)_{\mid M-C\left(r_{i}, q\right)}=f(q)_{\mid M-C\left(r_{i}, q\right)}$. One may assume, without loss of generality, that $r_{1}<\ldots<$ $r_{m}$ and that, because the interval is one-dimensional, no point of $\left[x_{0}, x_{1}\right]$ is included in more than two of the $I_{r_{i}}$. Thus pick any $q_{i} \in I_{r_{i}} \cap I_{r_{i+1}}$ for $i=1, \ldots, m-1$ and put $q_{0}=x_{0}$ and $q_{m}=x_{1}$. Let $C=\bigcup_{i=1, \ldots, m} C\left(r_{i}, q_{i-1}\right) \cup C\left(r_{i}, q_{i}\right)$ and observe that

$$
\begin{aligned}
f\left(x_{0}\right)_{\mid M-C} & =f\left(q_{0}\right)_{\mid M-C}=f\left(r_{1}\right)_{\mid M-C}=f\left(q_{1}\right)_{\mid M-C}=\cdots \\
& =f\left(q_{m-1}\right)_{\mid M-C}=f\left(r_{m}\right)_{\mid M-C}=f\left(q_{m}\right)_{\mid M-C}=f\left(x_{1}\right)_{\mid M-C} .
\end{aligned}
$$

Since $C$ is compact and $x_{0}, x_{1}$ were arbitrary, the proof is complete.

\section{A.2 Equivalence of continuity in the geometric sense and compact- open continuity}

To prove proposition 3, it will be helpful to use the bundles of Lorentz tensors over $M$ and degenerate metrics over $M \times \mathbb{R}$, denoted $\hat{L}(M)$ and $\hat{\Gamma}(M, \mathbb{R})$, respectively. The desired equivalence is essentially a corollary of the following (adapted from Munkres ([2000], p. 287, Theorem 46.11)).

Lemma 1. Let $X$ and $Y$ be topological spaces, and give the set of continuous functions from $X$ to $Y$, denoted $\mathcal{C}(X, Y)$, the $\left(C^{0}\right)$ compact-open topology. If $f: X \times Z \rightarrow Y$ is continuous, then so is the induced function $F: Z \rightarrow \mathcal{C}(X, Y)$ defined by the equation $(F(z))(x)=f(x, z)$. The converse holds if $X$ is locally compact ${ }^{37}$ and Hausdorff.

Proposition 3. A family of Lorentz metrics $\{\stackrel{t}{g}\}_{t \in \mathbb{R}}$ on $M$ is continuous in the geometric sense iff it is continuous in the $C^{0}$ compact-open topology. ${ }^{38}$

\footnotetext{
${ }^{37} \mathrm{~A}$ topological space is locally compact when each point has a compact neighborhood.

${ }^{38}$ Using jet bundles (Golubitsky and Guillemin [1973], ch. 2.2-2.3), this proposition may be generalized to any $C^{k}$ compact-open topology and families of Lorentz metrics parameterized by any smooth manifold.
} 
Proof. Let $\psi_{p}^{(t)}: M \rightarrow M \times \mathbb{R}$ be the embeddings that define the 5-dimensional metric $\Gamma_{a b}$, which corresponds to a cross-section $\tilde{\Gamma}$ of a bundle $\hat{\Gamma}(M, \mathbb{R})$ of 5 -dimensional metrics. The bundle homomorphism $\phi: \hat{\Gamma}(M, \mathbb{R}) \rightarrow \hat{L}(M)$ defined by $\phi\left(\tilde{\Gamma}_{\mid(p, t)}^{\prime}\right)=\left(\psi_{p}^{(t)}\right)^{*}\left(\Gamma_{a b}^{\prime}\right)$, for any local section $\tilde{\Gamma}^{\prime}$ at $(p, t) \in M \times \mathbb{R}$, is smooth because by definition the $\psi_{p}^{(t)}$ are jointly smooth in $p$ and $t$. Thus, if the family $\stackrel{t}{g}_{a b}$ is continuous in the geometric sense, $f=\phi \circ \tilde{\Gamma}: M \times \mathbb{R} \rightarrow \hat{L}(M)$ is continuous. Lemma 1 then entails that the map $F: \mathbb{R} \rightarrow \mathcal{C}(M, \hat{L}(M))$ defined by $F: t \mapsto \hat{g}$, where $\stackrel{t}{g}$ is the cross-section of $\hat{L}(M)$ corresponding to $\stackrel{t}{g}_{a b}$, is continuous when its range is given the $C^{0}$ compact-open topology.

Conversely, suppose that the family $\stackrel{t}{g}_{a b}$ is continuous in the $C^{0}$ compact-open topology, i.e., that $F: \mathbb{R} \rightarrow \mathcal{C}(M, \hat{L}(M))$ defined above is continuous. Since $M$ is locally compact and Hausdorff, lemma 1 entails that $f: M \times \mathbb{R} \rightarrow \hat{L}(M)$ is continuous. Thus $\left(\stackrel{t}{g}_{a b}\right)_{\mid p}$ is jointly continuous in $t$ and $p$. Note that $\Gamma^{a b}$ is continuous when, for any smooth field $\alpha_{a b}$ on $M \times \mathbb{R}, \alpha_{a b} \Gamma^{a b}$ is continuous. Now, for any $(p, t) \in M \times \mathbb{R},\left(\alpha_{a b} \Gamma^{a b}\right)_{\mid \psi^{(t)}(p)}=\left(\psi_{p}^{(t)}\right)^{*}\left(\alpha_{a b}\right)^{t} g^{a b} ;$ by assumption $\left(\psi_{p}^{(t)}\right)^{*}\left(\alpha_{a b}\right)$ is smooth; and $\stackrel{t}{g}^{a b}$ is continuous because its inverse is. Thus $\Gamma^{a b}$ is continuous, so $\Gamma_{a b}$ must be so as well by construction.

\section{A.3 Stable causality and the compact-open topologies}

The proof of proposition 4 uses proposition 5, but because the latter is a straightforward computation, the order of presentation follows that of the main text. First, a lemma is required allowing one to 'interpolate' between any spacetime and a chronology-violating region of Gödel spacetime. ${ }^{39}$

Lemma 2. Let $(M, g)$ and $\left(\mathbb{R}^{4}, g^{\prime}\right)$ be two spacetimes. For any open $S \subset M$ and any open $R \subset \mathbb{R}^{4}$ with compact closure, there is a spacetime $\left(M, g^{\prime}\right)$ such that $g_{\mid M-S}^{\prime}=g_{\mid M-S}$ and $g_{\mid R}^{\prime}$ is isometric to $g_{\mid U}^{\prime}$ for some $U \subset S .^{40}$

Proof. Pick a chart $(V, \varphi)$ of $M$ such that $V \subseteq S$ and $\varphi[V]$ is an open ball of radius 4, i.e., $\varphi[V]=B_{\mathbb{R}^{4}}(\overrightarrow{0}, 4)=\left\{\vec{x} \in \mathbb{R}^{4}:\|\vec{x}\|<4\right\}$, where $\|\cdot\|$ is the Euclidean norm on the coordinates $\vec{x} \in \mathbb{R}^{4}$. For brevity, define $A_{i}=\varphi^{-1}\left[B_{\mathbb{R}^{4}}(\overrightarrow{0}, i)\right]$ for $i=1,2,3$, and let $r$ be a scalar field on $V$ defined by $r_{\mid p}=\|\varphi(p)\|$. Finally, let $\psi: \mathbb{R}^{4} \rightarrow V$ be a diffeomorphism such that $\psi[R] \subseteq A_{1}$ and define $U=\psi[R]$.

Because all Lorentz metrics on $\mathbb{R}^{4}$ are homotopic (Finkelstein and Misner [1959]), $\psi_{*}\left(g^{\prime}\right)$ is homotopic to $g_{\mid V}$, considering $V$ as a submanifold. Thus there is some continuous function $f:[0,1] \rightarrow L(V)$ such that $f(0)=\psi_{*}\left(g^{\prime}\right)$ and $f(1)=g_{\mid V}$. One can then define the continuous Lorentz metric

$$
\gamma_{\mid p}= \begin{cases}g_{\mid p}, & p \in M-A_{3}, \\ f\left(r_{\mid p}-2\right)_{\mid p}, & p \in A_{3}-A_{2}, \\ {\left[\psi_{*}\left(g^{\prime}\right)\right]_{\mid p},} & p \in A_{2} .\end{cases}
$$

\footnotetext{
${ }^{39}$ For more on the properties of Gödel spacetime, see (Malament [2012], ch. 3.1).

${ }^{40}$ Following (Manchak [unpublished]), one can use this lemma to answer affirmatively a question posed by (Stein [1970], p. 594) about whether it is always possible to continuously deform a spacetime into one containing a closed timelike curve.
} 
In order to produce the desired smooth metric $g^{\prime}$, one can convolve $\gamma$ with an appropriate positive, symmetric mollifier on the region $V-A_{1}$. In more detail, define $w: \mathbb{R} \rightarrow \mathbb{R}$ to be the smooth function

$$
w(x)= \begin{cases}c e^{-1 /\left(1-x^{2}\right)}, & |x|<1 \\ 0, & |x| \geq 1\end{cases}
$$

where $c$ is a positive constant chosen so that $\int_{\mathbb{R}} w(x) d x=1$. Further, define $W: \mathbb{R}^{n} \times$ $[0, \infty) \rightarrow \mathbb{R}$ as the jointly smooth function $W(\vec{x}, \epsilon)=\epsilon^{-n} w(\|\vec{x}\| / \epsilon)$, where $W(\vec{x}, 0)=$ $\lim _{\delta \rightarrow 0} W(\vec{x}, \delta)$ is the Dirac delta, the convergence being understood in the distributional sense. Now, one can express $\gamma$ in terms of its matrix components $\gamma_{\alpha \beta}(\vec{x})$ determined by the chart $(V, \varphi)$, allowing one to define on $\varphi\left[V-A_{1}\right]$ for some fixed $\epsilon$ the new components

$$
\tilde{\gamma}_{\alpha \beta}(\vec{x})=\int_{\varphi\left[\operatorname{int}\left(V-A_{1}\right)\right]} W\left(\vec{x}-\vec{y}, e \epsilon c^{-1} w(\|\vec{x}\|-5 / 2)\right) \gamma_{\alpha \beta}(\vec{y}) d \vec{y},
$$

which are smooth on $\varphi\left[V-A_{1}\right] .{ }^{41}$ Moreover, for sufficiently small $\epsilon$, the $\tilde{\gamma}_{\alpha \beta}$ approximate the $\gamma_{\alpha \beta}$ arbitrarily well on $V-A_{1} \cdot{ }^{42}$ Therefore such $\tilde{\gamma}_{\alpha \beta}$ are the components of a smooth Lorentz metric $\tilde{\gamma}$ on $V-A_{1}$. Note that, in the integrand of eq. 8 the function $W$ becomes the Dirac delta for $\|\vec{x}\| \geq 7 / 2$ and $\|\vec{x}\| \leq 3 / 2$, so on the points of $V$ corresponding to these coordinate regions, $\tilde{\gamma}$ is equal to $g$ and $\psi_{*}\left(g^{\prime}\right)$, respectively. We can define at last

$$
g_{\mid p}^{\prime}= \begin{cases}g_{\mid p}, & p \in M-V, \\ \tilde{\gamma}_{\mid p}, & p \in V-A_{1}, \\ {\left[\psi_{*}\left(g^{\prime}\right)\right]_{\mid p},} & p \in A_{1} .\end{cases}
$$

By construction, $g_{\mid M-S}^{\prime}=g_{\mid M-S}$ since $M-S \subseteq M-V$ and $g_{\mid U}^{\prime}$ is isometric to $g_{\mid R}^{\prime}$ since $g_{\mid A_{1}}^{\prime}=\left[\psi_{*}\left(g^{\prime}\right)\right]_{\mid A_{1}}$ and $U=\psi[R] \subseteq A_{1}$.

Proposition 4. Chronology violating spacetimes are generic in $L(M)$ for every compactopen topology.

Proof. Every spacetime with a compact $M$ contains timelike curves (Hawking and Ellis [1973], p. 189, proposition 6.4.2), so suppose $M$ is non-compact. Select any neighborhood $N(g)$ of an arbitrary $g$, which must contain a set of the form $B_{k}(g, \epsilon ; h, C)$. Letting $S=$ $M-C$, by lemma 2 there is some $g^{\prime} \in N(g)$ such that $g_{\mid U}^{\prime}$ is isometric to a chronology violating region of Gödel spacetime for some $U \subset M-C$. By proposition 5 , there is an open neighborhood of $g^{\prime}$ consisting only of chronology violating metrics. Let $A_{k}\left(g, N(g), B_{k}\right)$ be the union of all such open neighborhoods determined by the choices of $g, N(g)$, and $B_{k}(g, \epsilon ; h, C)$, and consider $A=\bigcup_{g} \bigcup_{N(g)} \bigcup_{B_{k}} A_{k}\left(g, N(g), B_{k}\right)$. By construction, every neighborhood $N(g)$ of each $g$ contains an element of $A$, i.e., $A$ is dense in $L(M) ; A$ is open, being the union of open sets; and $A$ contains only chronology violating spacetimes. So by definition the chronology violating spacetimes are generic in $L(M)$.

\footnotetext{
${ }^{41}$ This follows essentially from theorem 2.6 of Oden and Reddy ([1976], pp. 48-49).

42 Theorem 2.7 of (Oden and Reddy [1976], p. 49) shows that, for the case where the integrand contains $W(\vec{x}-\vec{y}, \delta)$ with a fixed $\delta$, the analog of eq. 8 would converge to $\gamma_{\alpha \beta}$ as $\delta \rightarrow 0$ in $L^{p}\left(\varphi\left[\operatorname{int}\left(V-A_{1}\right)\right]\right)$-norm. As before (cf. footnote 41 ), allowing $\delta$ to smoothly vary introduces no new complications.
} 
Proposition 5. If the spacetime $(M, g)$ contains a closed timelike curve, then $g$ is stably chronology violating in every compact-open topology.

Proof. Fix any Riemannian metric $h_{a b}$ and note that one can write $g_{a b}=h_{a m} \mu^{m} h_{b n} \mu^{m}-h_{a b}$ for some smooth vector field $\mu^{a}$ (Hawking and Ellis [1973], p. 39). One can thus express that $\gamma: I \rightarrow M$ is a closed $g$-timelike curve with unit tangent vector $\xi^{a}$ as the condition that $\left|h_{a b} \xi^{a} \mu^{b}\right|_{\mid \gamma[I]}>\left(h_{a b} \xi^{a} \xi^{b}\right)_{\mid \gamma[I]}^{1 / 2}$. Pick

$$
\epsilon=\inf _{\gamma[I]} \min \left\{1,\left(\frac{\left|h_{a b} \xi^{a} \mu^{b}\right|}{\left(h_{a b} \xi^{a} \xi^{b}\right)^{1 / 2}}-1\right)^{2}\right\}
$$

and consider any $g^{\prime} \in B_{0}(g, \epsilon ; h, C)$, where $\gamma[I] \subseteq C$. Writing $g_{a b}^{\prime}=h_{a m} \mu^{\prime m} h_{b n} \mu^{\prime m}-h_{a b}, \gamma$ is $g^{\prime}$-timelike just in case $\left|h_{a b} \xi^{a} \mu^{\prime b}\right|_{\mid \gamma[I]}>\left(h_{a b} \xi^{a} \xi^{b}\right)_{\mid \gamma[I]}^{1 / 2}$. Now, one can calculate that

$h^{a m} h^{b n}\left(g_{a b}-g_{a b}^{\prime}\right)\left(g_{m n}-g_{m n}^{\prime}\right)=h_{a m} h_{b n}\left(\mu^{a} \mu^{b}-\mu^{\prime a} \mu^{\prime b}\right)\left(\mu^{m} \mu^{n}-\mu^{\prime m} \mu^{\prime n}\right)=\left[h_{a b}\left(\mu^{a}-\mu^{\prime a}\right)\left(\mu^{b}-\mu^{\prime b}\right)\right]^{2}$,

so putting $\eta^{a}=\mu^{\prime a}-\mu^{a}$ yields that $\sup _{C} h_{a b} \eta^{a} \eta^{b}<\epsilon$. (The remaining calculations involve fields defined on $\gamma[I]$, so the subscript indicating as much will be omitted.) It follows from this inequality and the Cauchy-Schwartz inequality that

$$
\left|h_{a b} \xi^{a} \eta^{b}\right| \leq\left(h_{a b} \xi^{a} \xi^{b}\right)^{1 / 2}\left(h_{a b} \eta^{a} \eta^{b}\right)^{1 / 2}<\left(\epsilon h_{a b} \xi^{a} \xi^{b}\right)^{1 / 2} \leq\left(h_{a b} \xi^{a} \xi^{b}\right)^{1 / 2}
$$

where the last inequality uses the fact that, by definition, $\epsilon \leq 1$. Then the reverse triangle inequality entails that

$$
\left|h_{a b} \xi^{a} \mu^{\prime b}\right|=\left|h_{a b} \xi^{a}\left(\mu^{b}+\eta^{b}\right)\right| \geq|| h_{a b} \xi^{a} \mu^{b}|-| h_{a b} \xi^{a} \eta^{b}||=\left|h_{a b} \xi^{a} \mu^{b}\right|-\left|h_{a b} \xi^{a} \eta^{b}\right|,
$$

where the last equality follows since $\left|h_{a b} \xi^{a} \mu^{b}\right|>\left(h_{a b} \xi^{a} \xi^{b}\right)^{1 / 2}>\left|h_{a b} \xi^{a} \eta^{b}\right|$ by the hypothesis and equation 9. Applying this equation again along with the definition of $\epsilon$ yields that

$\left|h_{a b} \xi^{a} \mu^{\prime b}\right|>\left|h_{a b} \xi^{a} \mu^{b}\right|-\left(\epsilon h_{a b} \xi^{a} \xi^{b}\right)^{1 / 2} \geq\left|h_{a b} \xi^{a} \mu^{b}\right|-\left(h_{a b} \xi^{a} \xi^{b}\right)^{1 / 2}\left|\frac{\left|h_{a b} \xi^{a} \mu^{b}\right|}{\left(h_{a b} \xi^{a} \xi^{b}\right)^{1 / 2}}-1\right|=\left(h_{a b} \xi^{a} \xi^{b}\right)^{1 / 2}$.

Thus $\gamma$ is $g^{\prime}$-timelike, but $g^{\prime}$ was arbitrary so each element of $B_{0}(g, \epsilon ; h, C)$ contains a closed timelike curve. Since $B_{0}(g, \epsilon ; h, C)$ is open in every $C^{k}$ compact-open topology, $g$ must be stably chronology violating in each.

Corollary 2. Suppose $\mathcal{T}_{M}$ is a topology on $L(M)$, for any $M$, that makes continuous all the one-parameter families continuous in the geometric sense. Then, for any $g \in L(M)$ there is no compact $C \subseteq M$ such that $g_{\mid \operatorname{int}(C)}$ is stably causal in the topology $\mathcal{T}_{\operatorname{int}(C)}$ on $L(\operatorname{int}(C))$.

Proof. Consider an arbitrary $g \in L(M)$ and a compact $C \subseteq M$. By corollary $1, g_{\mid \operatorname{int}(C)}$ is not stably causal in the $C^{0}$ compact-open topology on $L(\operatorname{int}(C))$. But if $\mathcal{T}_{M}$ makes continuous all the one-parameter families continuous in the geometric sense, it follows from proposition 3 that it is no finer that the $C^{0}$ compact-open topology, hence $g_{\mid} \operatorname{int}(C)$ is not stably causal in the topology $\mathcal{T}_{\operatorname{int}(C)}$ on $L(\operatorname{int}(C))$ either. 


\section{References}

Beem, J. K., Ehrlich, P. E., and Easley, K. L. [1996]: Global Lorentzian Geometry, 2nd edn, New York: Marcel Dekker.

Bombelli, L., and Meyer, D. A. [1989]: 'The Origin of Lorentzian Geometry', Physics Letters A, 141, pp. 226-8.

Earman, J. [1995]: Bangs, Crunches, Whimpers, and Shrieks, New York: Oxford University Press.

Finkelstein, D. and Misner, C. W. [1959]: 'Some New Conservation Laws', Annals of Physics, 6, pp. 230-43.

Fletcher, S. C. [2012]: 'What Counts as a Newtonian System? The View from Norton's Dome', European Journal for Philosophy of Science, 2(3), pp. 275-97.

Geroch, R. [1969]: 'Limits of Spacetimes', Communications in Mathematical Physics, 13, pp. 180-93.

Geroch, R. [1970]: 'Singularities', in M. Carmeli, S. I. Fickler, and L. Witten (eds), Relativity, New York: Plenum, pp. 259-91.

Geroch, R. [1971]: 'General Relativity in the Large', General Relativity and Gravitation, 2(1), pp. 61-74.

Geroch, R. [1985]: Mathematical Physics, Chicago: University of Chicago Press.

Golubitsky, M. and Guillemin, V. [1973]: Stable Mappings and their Singularities, New York: Springer.

Halvorson, H. [2012]: 'What Scientific Theories Could Not Be', Philosophy of Science, 79(2), pp. 183-206.

Hawking, S. W. [1971]: 'Stable and Generic Properties in General Relativity', General Relativity and Gravitation, 1(4), pp. 393-400.

Hawking, S. W. and Ellis, G. F. R. [1973] The Large Scale Structure of Space-time, Cambridge: Cambridge University Press.

Henson, J. [2009]: 'The Causal Set Approach to Quantum Gravity', in D. Oriti (ed.), Approaches to Quantum Gravity, Cambridge: Cambridge University Press, pp. 393-413.

Henson, J. [2012]: 'Causal Sets: Discreteness without Symmetry Breaking', in J. Murugan, A. Weltman and G. F. R. Ellis (eds), Foundations of Space and Time: Reflections on Quantum Gravity, Cambridge: Cambridge University Press, pp. 385-409.

Lerner, D. E. [1972]: The Space of Lorentz Metrics on a Non-Compact Manifold, PhD dissertation, University of Pittsburgh. 
Lerner, D. E. [1973]: 'The Space of Lorentz Metrics', Communications in Mathematical Physics, 32, pp. 19-38.

Lerner, D., and Porter, J. R. [1974]: 'Weak gravitational fields', Journal of Mathematical Physics, 15, pp. 1413-15.

Lewis, D. [1973]: Counterfactuals, Oxford: Blackwell.

Lewis, D. [1986]: Philosophical Papers, vol. II, Oxford: Oxford University Press.

Malament, D. B. [1986]: 'Newtonian Gravity, Limits, and the Geometry of Space', in R. G. Colodny (ed.), From Quarks to Quasars, Pittsburgh: University of Pittsburgh Press, pp. $181-201$.

Malament, D. B. [2012]: Topics in the Foundations of General Relativity and Newtonian Gravitation Theory, Chicago: University of Chicago Press.

Manchak, J. [2011]: 'What Is a Physically Reasonable Spacetime?', Philosophy of Science, 78, pp. $410-20$.

Manchak, J. [unpublished]: 'A Note on "Nearby" Universes'.

Minguzzi, E. [2009]: 'Chronological Spacetimes without Lightlike Lines are Stably Causal', Communications in Mathematical Physics, 288, pp. 801-19.

Munkres, J. R. [2000]: Topology, 2nd edn, Upper Saddle River, NJ: Pearson Prentice Hall.

Noldus, J. [2002]: 'A New Topology on the Space of Lorentzian Metrics on a Fixed Manifold', Classical and Quantum Gravity, 19, pp. 6075-107.

Norton, J. D. [2008]: 'The Dome: An Unexpectedly Simple Failure of Determinism', Philosophy of Science, 75, pp. 786-98.

Oden, J. T. and Reddy, J. N. [1976]: An Introduction to the Mathematical Theory of Finite Elements, New York: Wiley.

Schroeter, F. [2004]: 'Reflective Equilibrium and Antitheory', Nô̂s, 38(1), pp. 110-34.

Smeenk, C. and Wüthrich, C. [2011]: 'Time Travel and Time Machines', in C. Callender (ed.), The Oxford Handbook of Philosophy of Time, Oxford: Oxford University Press, pp. 577-630.

Stein, H. [1970]: 'On the Paradoxical Time-Structures of Gödel', Philosophy of Science, 37(4), pp. 589-601. 